\title{
Aberration Corrected Electron Microscopy Enhanced for Lower Accelerating Voltages
}

\author{
Takaki Ishikawa $^{1}$, Eiji Okunishi ${ }^{1}$, Toshikatsu Kaneyama $^{1}$, Yukihito Kondo $^{1}$, Syo Matsumura $^{2}$ \\ 1. JEOL Ltd., 3-1-2 Musashino Akishima Tokyo 196-8558 Japan. \\ 2. Research laboratory of high-voltage electron microscope, Kyushu University, Motooka 744 Nishi-ku \\ Fukuoka 819-0395 Japan.
}

Radiation damage by incident electron beam becomes a serious problem in chemical analysis by EDS and/or EELS, since the dose for these analysis requires more than that for imaging. Low acceleration voltage (here in after we call "low $\mathrm{kV}$ " in this paper) is effective to solve the problem, since it reduces the specimen damage of materials composed of light elements such as carbon materials. The dedicated low $\mathrm{kV}$ microscopes for the purpose have been developed on the special projects $[1,2]$. However, we can make an adequate tuning for a low $\mathrm{kV}$ such as 80,60 and $30 \mathrm{kV}$ on the aberration corrected 200 or $300 \mathrm{kV}$ microscopes (ex. JEOL JEM-ARM200F). In this paper, we explore the analytical capabilities of JEM-ARM200F for the performances in low $\mathrm{kV}$.

In low $\mathrm{kV}$, an obstacle to make atomic resolution image or analysis is an image blur $(\delta)$, determined with a chromatic aberration coefficient $(\mathrm{Cc})$, an energy spread of an electron source (dE) and primary electron energy (Eo), as $\delta=\mathrm{Cc} * \mathrm{dE} / \mathrm{Eo}$. As Eo decreases in low $\mathrm{kV}$, the blur $(\delta)$ becomes larger. Therefore, small $\mathrm{Cc}^{*} \mathrm{dE}$ is essential to make a small probe. The Cc also decreases as Eo decreases, since the saturation of the magnetic objective lens released as the required excitation of the lens reduces. With an electron source from cold field emission gun (CFEG), dE is 0.3-0.4 eV. Figure 1(a) and 1(b) plot the probe diameters (in D59) at $60 \mathrm{kV}$ and $200 \mathrm{kV}$ with thermal field emission gun (TFEG) and CFEG, and with A5 aberration correction or not, depending on the convergence angle. The D59 is a probe diameter which includes $59 \%$ of the total probe current. The total chromatic aberration coefficients $(\mathrm{Cc})$ for this calculation were $2.36 \mathrm{~mm}$ for $200 \mathrm{kV}$ and $1.41 \mathrm{~mm}$ for $60 \mathrm{kV}$, which are calculated sum of $\mathrm{Cc}$ for the objective lens with HRP (analytical poles allowing highly sensitive dual EDS detectors system) and the aberration correction system optics. Since the specimen damage decreases in low $\mathrm{kV}$, we can increase the allowable current of analytical probe, resulting in better analytical sensitivity. In addition to this, the ionization cross section increases in low $\mathrm{kV}$. It also enhances the sensitivity.

To obtain more probe-current keeping the probe size small, convergence angle must increase. Namely, it is essential to reduce the higher order geometrical aberration such as sixth fold astigmatism (A5). As shown in Fig. 1, without the correction of A5, the allowable aperture size starts to increase rapidly at 40 mrad. Therefore, it is effective to correct A5 when we use the CFEG. Figures 2(a) and 2(b) show Ronchigrams obtained with JEM-ARM200F equipped with CFEG and the higher order geometrical aberration (DCOR CEOS Gmbh). The flat phase regions, which are generally 25-30 mrad with no A5 correction, clearly increase to be 55-60 mrad in these Ronchigrams. Thus, the probe current can be increased. On the other hand, with highly sensitive detectors are also sought after for the analysis of beam sensitive materials. The dual SDD system, developed recently [3], dramatically raises the detection solid angle of fluorescence X-ray from a sample to be $>1.7$ sr. Figure 3 shows the obtained elemental maps from $\mathrm{SrTiO}_{3}$ with JEM-ARM200F equipped with the dual SDD system, HR type objective lens and CFEG at $200 \mathrm{kV}$. The acquisition conditions for these maps are probe size $=90 \mathrm{pm}$, 
probe current $=24 \mathrm{pA}$, number of pixels $=128 \times 128$, dwell time for each pixel $=10 \mu \mathrm{sec}$ and number of integrated frames $=1000$. Each element is clearly separated on these maps with rather short time and rather small probe current, which allows us to observe an atomic level high resolution STEM image.

Thus, with employment of dual SDD system, low $\mathrm{kV}$ and higher order aberration correction system, the analytical sensitivity is greatly improved and it greatly lowers the threshold of the analysis of beam sensitive materials.

\section{References:}

[1] Suenaga project, "Low-voltage TEM/STEM for atomic level characterization of soft matters", CREST (2006-2012) Japan Science \& Technology Agency.

[2] Salve project, Sub-Angstrom Low-Voltage Electron Microscopy (SALVE) I-II project (2008 ).

[3] S Kawai et al, Microscopy and Microanalysis 20 (S3) (2014) p.1150.
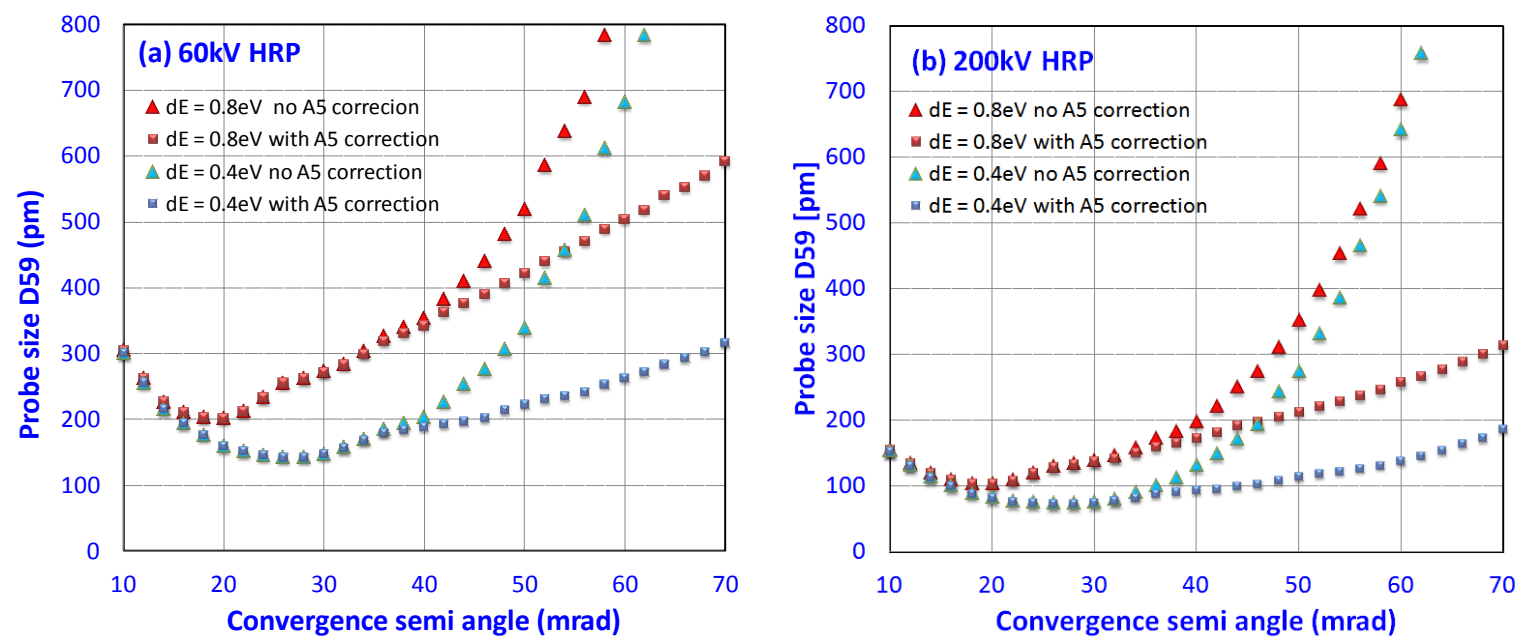

Figure 1 Probe diameter depending on convergence semi angle with CFEG, TFEG, A5 correction.

(a) at $60 \mathrm{kV}$, (b) at $200 \mathrm{kV}$
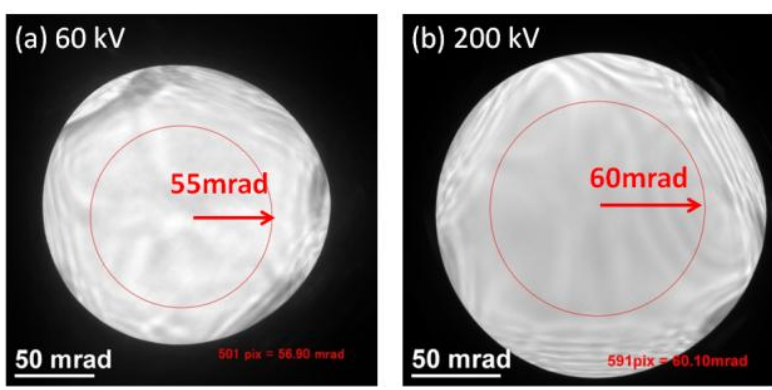

Figure 2 Observed Ronchigram from amorphous carbon film. The microscope equippec with higher order aberration corrector and CFEG. (a) at $60 \mathrm{kV}$, (b) at $200 \mathrm{kV}$
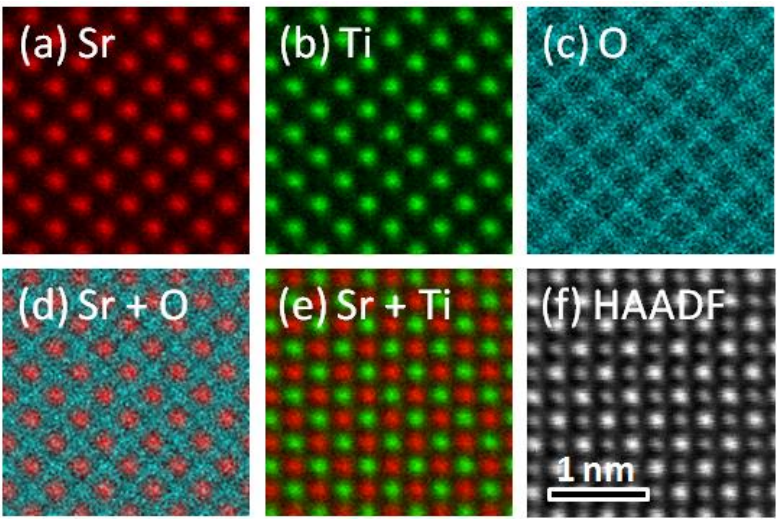

Figure 3 Elemental maps with dual SDD system from $\mathrm{SrTiO}_{3}$.

(a) $\mathrm{Sr}$, (b) $\mathrm{Ti}$, (c) $\mathrm{O}$, (d) $\mathrm{Sr}+\mathrm{O}$, (e) $\mathrm{Sr}+\mathrm{Ti}$, (f) HAADF STEM image 